\title{
Giant retroperitoneal mature cystic teratoma in an infant: a case report
}

\begin{abstract}
We describe an unusual case of a 6 weeks old male baby, referred to our hospital due to a large mass in his abdomen. Radiological work-up revealed a large retroperitoneal mass. Exploratory laparotomy with tumor resection was performed. Histopathological examination confirmed a mature cystic teratoma. The baby is doing well on follow up.
\end{abstract}

Keywords: retroperitoneum, teratoma, laparotomy
Volume 5 Issue 7 - 2016

\author{
Venkatesh M Annigeri,' Bhuvanesh A, ${ }^{2}$ \\ Bahubali D Gadgade, ${ }^{3}$ Anil B Halgeri ${ }^{1}$ \\ 'Professor, Department of Paediatric Surgery, Urology SDM \\ College of Medical Sciences and Hospital, India \\ ${ }^{2}$ Associate Professor, Department of Paediatric Surgery, Urology \\ SDM College of Medical Sciences and Hospital, India \\ ${ }^{3}$ Assistant Professor, Department of Paediatric Surgery, Urology \\ SDM College of Medical Sciences and Hospital, India
}

Correspondence: Venkatesh M Annigeri MS, MCh, Professor, Department of Paediatric surgery, SDM College of Medical Sciences and Hospital, Sattur, Dharwad, Karnataka, India, Tel 91836-2477802, Fax 91836-246।65।,

Email drvenkateshpgi@rediff.com

Received: November 19, 2016 | Published: December 06, 2016

\section{Introduction}

Teratomas are congenital tumors comprising of tissues, arising from pluripotent embryonic cells. They are frequently seen in gonads. The involvement of extragonadal sites in decreasing order of frequency are anterior mediastinum, retroperitoneum, pre sacrococcygeal areas, pineal and other intracranial sites. ${ }^{1}$ Retroperitoneal teratoma (RPT) is a very rare germ cell tumors in children and comprises $3.5-4 \%$ of all germ cell tumors and $1-11 \%$ of primary retroperitoneal neoplasms. ${ }^{2,3}$

\section{Case report}

A 6 weeks old male infant, born by normal vaginal delivery at home, presented to our hospital with progressively increasing abdominal mass of 10 days duration, associated with poor feeding. Antenatal care was unsupervised. There was no history of fever, weight loss, bowel or urinary complaints. Post natal period was uneventful. On examination the abdomen was distended, a large firm mass occupying the central and left upper abdomen measuring about $15 \times 12 \times 10 \mathrm{~cm}$ in maximum dimensions. No tenderness on palpation or overlying skin changes. The rest of general examination was unremarkable. Routine blood tests and urine analysis were all within normal limits. The serum levels of alpha-fetoprotein (AFP) was normal. A plain abdominal X-ray showed a well defined round radio-dense mass seen projecting at the left side of the abdomen, mass effect was evident marked displacement of the bowel loops peripherally (Figure 1A). Ultrasound of abdomen showed a large heterogeneous mass located on the left side associated with areas of cystic components. Contrast enhanced computed tomography (CECT) scan of the abdomen and pelvis demonstrates a large retroperitoneal heterogeneous mass occupying predominantly the left suprarenal region, measuring about $12 \times 10 \times 8 \mathrm{~cm}$ with two clumps of calcification, fatty tissue, and cystic contents. The mass crossing the midline was displacing the aorta and Inferior Vena Cava to the contralateral side and the left kidney posterior-inferiorly (Figure 1B\&1C). The rest of abdominal and pelvic organs were within normal limits.
The baby was planned for surgical excision of the mass. Exploratory transverse laparotomy was performed. On laparotomy a large complex, solid and cystic mass, with well circumscribed smooth border was seen. The pancreas was flattened and stretched over the cranial portion of the tumor (Figure 2A). There was no invasion to the aorta or inferior vena cava. The tumor measured $12 \times 10 \times 8 \mathrm{~cm}$ and weighted 700 gms. Cut section of the mass revealed multilocular cystic spaces, whitish grey wall scattered adipose tissue collection and areas of calcifications. The entire tumor was then excised and sent for histopathological reporting (Figure 2B). Histopathology confirmed the diagnosis of a mature cystic teratoma, benign in nature, with no malignant cells. The final diagnosis was made to be mature cystic teratoma of the retroperitonium. Post operative course was uneventful and baby was discharged on post operative day 8 in good and stable condition. On follow up, baby is doing well with adequate weight gain.

\section{Discussion}

Retroperitoneal teratomas are the third most common primary retroperitoneal tumours in the paediatric population after neuroblastroma and Wilms tumour. ${ }^{4}$ Teratomas arise from germ cells that fail to mature normally in the gonadal locations. These totipotent cells can differentiate into tissue components representing derivatives of mesoderm, ectoderm and endoderm. The distribution of teratomas listed in order of decreasing frequency is: ovaries, testes, anterior mediastinum, retroperitoneal space, pre sacro-coccygeal areas, pineal and other intracranial sites, neck and abdominal viscera other than gonads. The developmental migratory properties of germ cells would explain teratomas in these extragonadal sites, which generally occur along midline structures. ${ }^{5,6}$ Majority of the tumors are benign, situated on the left side and para renal in location; occasional lesions are bilateral. Occurrence of RPT bimodal, with peaks in the first 6 months of life and in early adulthood. Additionally, the incidence of RPT in females is twice that in males. RPT often remain asymptomatic due to their location, and are usually identified only after they have grown to 
huge proportions. ${ }^{7}$ Patients usually present with abdominal distension or a palpable mass like in our case.

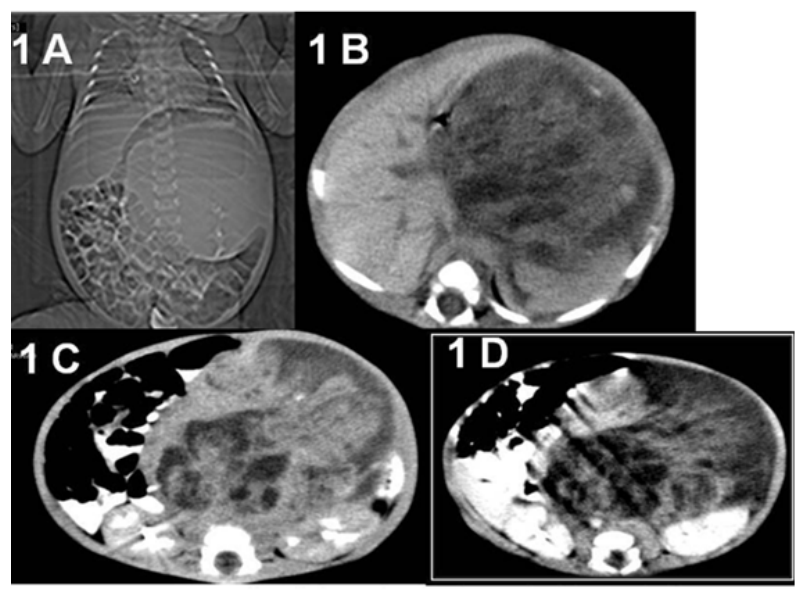

Figure I Images of computed tomography abdomen and pelvis.

IA: CT topographic image showing large left sided abdominal mass displacing the bowel loops with internal calcification.

IB: Plain CT axial image showing a large heterogeneous left suprarenal retroperitoneal mass with internal calcification.

IC: Contrast CT axial image showing a large heterogeneously enhancing left suprarenal retroperitoneal mass with enhancing soft tissue components and non enhancing cystic and necrotic parts.

ID: Contrast CT axial image showing a large heterogeneously enhancing left suprarenal retroperitoneal mass crossing midline, displacing aorta without encasing it. Left kidney is displaced inferiorly and compressed against the posterior abdominal wall.
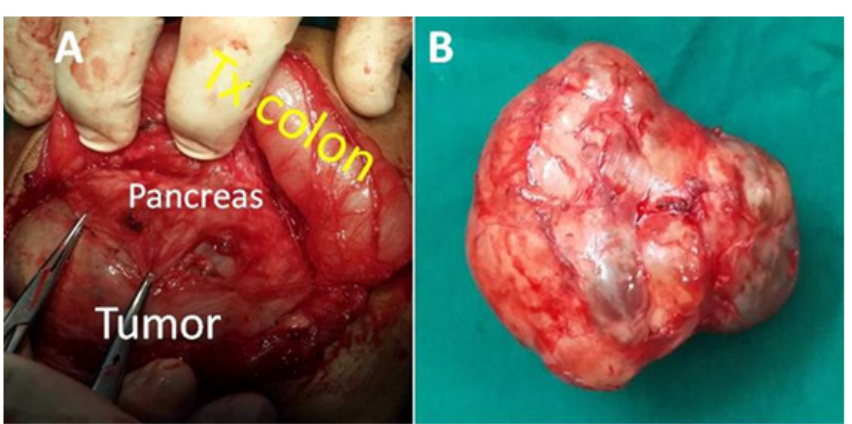

Figure 2 Surgical findings upon laparotomy.

2A: On laparotomy a large complex solid and cystic mass with well circumscribed smooth borders. The pancreas is flattened and stretched over the tumor.

2B: Gross image of the resected tumor showed a large complex solid and cystic mass measuring about $12 \times 10 \times 8 \mathrm{~cm}$ and weighting 700 grams.

Preoperatively, the diagnosis can be established by its characteristic appearance on radiologic evaluation and biochemical study. Radiological manifestations of teratomas are presence of calcification, teeth and fat. Calcification is seen in $50-60 \%$ of the cases and is often helpful in making diagnosis preoperatively. ${ }^{8}$ Diagnosis can be possible with the help of plain radiography, ultrasound abdomen and CECT or magnetic resonance image (MRI) of abdomen. Ultrasound of the abdomen is usually the first imaging modality. CECT or MRI can identify various components of these tumors, including bone, soft-tissue density structures, adipose tissue, and sebaceous and serous-type fluids. These imaging studies can also display the precise location, morphology and adjacent structures of the tumor, which provide better preoperative planning and increased likelihood of complete removal of the tumor with less iatrogenic damage . The main step of therapy for RPT is complete surgical resection and the prognosis for children with RPT depends primarily upon the adequacy of surgical resection. The most important aspect of the excision is to dissect the tumour from renal and other major vessels, which are invariably stretched out over the lesion. Prognosis is generally good and curative if the tumour is completely removed. Unresectable lesions, immature teratomas and endodermal sinus tumours have a comparatively worse prognosis, and may warrant aggressive chemotherapy due to their metastatic nature. ${ }^{4,9}$ Lastly the single most important factor in prognosis of RPT is complete removal which must be tried in every case of RPT irrespective of its size. The prognosis is excellent for benign RPT if complete resection can be accomplished.

\section{Acknowledgements}

None.

\section{Conflicts of interest}

The authors declare no conflict of interest.

\section{Funding}

None.

\section{References}

1. Engel RM, Elkins RC, Fletcher BD. Retroperitoneal teratoma. Review of the literature and presentation of an unusual case. Cancer 1968;22(5):1068-1073.

2. Grosfeld JL, Ballantine TV, Lowe D, et al. Benign and malignant teratomas in children:Analysis of 85 patients. Surgery. 1976;80(3):297305 .

3. Nguyen CT, Kratovil T, Edwards MJ. Retroperitoneal teratoma presenting as an abscess in childhood. J Pediatr Surg. 2007;42(11):2123.

4. Gatcombe, HG, Assikis V, Kooby D. Primary retroperitoneal teratomas:a review of the literature. J Surg Oncol. 2004;86(2):107-113.

5. Horton Z, Schlatter M, Schultz S. Pediatric germ cell tumors. Surg Oncol. 2007;16(3):205-213.

6. McKenney JK, Heerema-McKenney A, Rouse RV. Extragonadal germ cell tumors:a review with emphasis on pathologic features, clinical prognostic variables, and differential diagnostic considerations. Adv Anat Pathol. 2007;14(2):69-92.

7. Grosfeld JL, Ballantine TV, Lowe D, et al. Benign and malignant teratomas in children:Analysis of 85 Patients. Surgery. 1976;80(3):297305.

8. Bruneton JN, Diard F, Drouillard JP, et al. Primary retroperitoneal teratoma in adults. Radiology. 1980;134:613-616.

9. Ding Q, Zhu Y, Liang W. Adrenal teratoma:a case report and literature review. Chinese J Androl. 2008;10:40-43. 